\title{
ETHICAL CONSIDERATIONS IN ANIMAL RESEARCH: THE PRINCIPLE OF 3R'S
}

\author{
Lorenza díaz ${ }^{1}$, Elena Zambrano ${ }^{1}$, María E. Flores ${ }^{1,2}$, Mariela Contreras $^{1}$, Jose C. Crispín ${ }^{1}$, \\ Gabriela Alemán ${ }^{1}$, César Bravo ${ }^{1}$, Alejandra Armenta ${ }^{1}$, Victor J. Valdés ${ }^{1,3}$, Armando Tovar ${ }^{1}$, \\ Gerardo Gamba ${ }^{1,2}$, Jorge Barrios-Payán ${ }^{1}$, and Norma A. Bobadilla ${ }^{1,2 *}$ \\ ${ }^{1}$ The Institutional Animal Care and Use Committee, Instituto Nacional de Ciencias Médicas y Nutrición Salvador \\ Zubirán, Mexico City; ${ }^{2}$ Instituto de Investigaciones Biomédicas, Universidad Nacional Autónoma de México, \\ Mexico City; ${ }^{3}$ Instituto de Fisiología Celular, Universidad Nacional Autónoma de México, Mexico City, Mexico
}

\begin{abstract}
In the last century, progress in the knowledge of human diseases, their diagnosis and treatment have grown exponentially, due in large part to the introduction and use of laboratory animals. Along with this important progress, the need to provide training and guidance to the scientific community in all aspects related to the proper use of experimental animals has been indispensable. Animal research committees play a primary role in evaluating experimental research protocols, from their feasibility to the rational use of animals, but above all in seeking animal welfare. The Institutional Committee for the Care and Use of Animals (IACUC) has endeavored to share several relevant aspects in conducting research with laboratory animals. Here, we present and discuss the topics that we consider of utmost importance to take in the account during the design of any experimental research protocol, so we invite researchers, technicians, and undergraduate and graduate students to dive into the fascinating subject of proper animal care and use for experimentation. The main intention of these contributions is to sensitize users of laboratory animals for the proper and rational use of them in experimental research, as well as to disseminate the permitted and unpermitted procedures in laboratory animals. In the first part, the significance of experimental research, the main functions of IACUC, and the principle of the three R's (replacement, reduction, and refinement) are addressed.
\end{abstract} (REV INVEST CLIN. 2021;73(4):199-209)

Key words: Institutional Animal Care and Use Committee. Reduction. Refinement. Replacement.

\section{SIGNIFICANCE OF ANIMAL RESEARCH}

Biological processes are complex, interactive, and subjected to continuous influence by environmental factors. To understand the fundamental processes that maintain and integrate all the cells in the body, as well as the pathologies that emerge when homeostasis is altered, it is necessary to develop and study controlled and reproducible situations. Once that is done, it is possible to perform experimental interventions and observe how the system responds. While much can be studied in vitro, the integrated nature of

\section{*Corresponding author:}

Norma A. Bobadilla

E-mail: norma.bobadillas@incmnsz.mx/ nab@biomedicas.unam.mx

Received for publication: 28-07-2020

Approved for publication: 07-09-2020

DOI: $10.24875 / R I C .20000380$

0034-8376 / (c) 2020 Revista de Investigación Clínica. Published by Permanyer. This is an open access article under the CC BY-NC-ND license (http://creativecommons.org/licenses/by-nc-nd/4.0/). 
the mammalian organism requires specific studies to be conducted in living animals. Most of the experimental animal research is directed to improve health, life span, and quality of life of humans ${ }^{1}$. In addition, information from many studies designed to address human health also improves the quality of life of companion and agricultural animal species. Experimental animal studies in veterinary medicine represent about $5 \%$ of the total number of animals involved in experimental research².

Animals play multiple roles in daily human life, providing food, clothing, work, transportation, and human company (pets), in addition to their use in biomedical research. It is important to note that the number of animals studied in experimental settings represents $<0.003 \%$, which is 3 in every 100,000 compared to the number of animals consumed for food ${ }^{3}$. Nevertheless, the ethics of animal experimentation represents a topic that rightly generates controversy and discussion.

The research framework that has evolved over the last two decades has resulted in the presence of strong institutional oversight to ensure high ethical standards for all animal research ${ }^{4}$. Examples of poor conduct may still exist, but they are few, and we are working to avoid them. The majority of researchers conduct valuable animal experimental work in an ethical fashion that includes all precautions and Institutional and governmental oversight to maintain high standards to guarantee animal welfare. As a result, valuable information has emerged that enables the biomedical profession to prevent, mitigate, and even eradicate many human and animal diseases, in addition, to understand basic processes in physiology. These advances include vaccines and powerful medications for conditions such as: diabetes, obesity, cancer, hypertension, chronic kidney injury, brain, cardiovascular, and pulmonary diseases, among others, which affect both animals and humans. The physiological or pathophysiological processes that lack animal models for study are the less comprehended of all. For instance, the physiological or molecular processes involved in loving or missing somebody are completely unknown.

The extent to which research is carried out in different animal species depends on the similarities and differences with humans as well as the availability of resources and facilities. There are many reasons why animal studies are essential to medical progress. All mammals descend from common ancestors and all species have a similar set of organs that function in essentially the same way ${ }^{5}$. Animals and humans are biologically similar; therefore, they are susceptible to similar pathologies. Importantly, the World Medical Association Declaration of Helsinki states that all human clinical trials must be preceded by animal testing $^{6}$, recognizing that animal models, including nonhuman primates, are important for understanding, diagnosing, and developing treatments for safe and effective use in human diseases ${ }^{7}$.

Animals are biologically appropriate research subjects because their physiology is generally similar to human beings. In particular, rodents (with some exceptions such as guinea pigs) are altricial and polytocous species bearing large litters and born after a relatively short period of gestation in an immature state (blind, deaf, unable to regulate body temperature, and with limited motor coordination), requiring considerable maternal care in the immediate postnatal period to regulate basic neonatal functions ${ }^{8}$. Thus, choice of animal species to study depends on the biomedical question being asked. A variety of species can usually provide valuable data since comparative physiology gives the opportunity to observe similarities and differences among the systems.

From the practical point of view, many experimental species studied, such as rodents, often make better research subjects than human beings due to their shorter life cycles, easy handling accessible animal housing, etc 5 . Under laboratory conditions, rodents have a lifespan of 2-3 years, so researchers can conduct studies throughout their live cycle or even for several generations. This consideration is especially important when studying aging and transgenerational disease transmission. Animal studies are considerably more controllable than human clinical studies. In addition, rodent and other animal studies often provide a greater depth of mechanistic understanding due to the ability to conduct biopsies and fresh tissue retrieval for in vitro analysis, for example, molecular biology and histology 9 . Due to their shorter lifespan, life-course results are obtained much more quickly in animal models, which is especially important when studying aging and transgenerational disease transmission. 
The reproducibility and independent confirmation, which are requirements of scientific certainty, are generally easier to achieve in animal studies. Clinical trials are carefully designed to improve human health: however, in addition to the length of time required to obtain enough data, clinical trials have to deal with multiple confounders related to the subject's socioeconomic and health status, which not only complicate the analysis and interpretation but may also limit the usefulness of findings to determine the mechanisms involved. Human epidemiology can provide clues regarding disease associations but do not in themselves provide evidence of causation. All of these limitations in human epidemiology and clinical investigation make it essential that initial research be carried out by the use of experimental models of human diseases, aided by in vitro systems, such as cell cultures and by computational analysis as long as they are accessible ${ }^{10}$.

Living systems like human beings are extremely complex and while non-animal methods play an important role in biomedical research, they cannot be replaced by animal studies. In this sense, most powerful computers do not effectively substitute experimental models, even when scientists depend on computers to process data collected from in silico simulations ${ }^{11}$.

There are many medical examples of benefits from animal research because without the information obtained many options of treatment would not be available. Perhaps one of the best examples comes from the development of an effective oral vaccine to combat polio by Albert Sabin, who practically eradicated this disease after several experimentation rounds using approximately 9000 monkeys, 150 chimpanzees, and 133 human volunteers ${ }^{12}$. The contemporary example would be the desperate search worldwide to produce a COVID-19 vaccine. In relation to this infection, the emerging evidence that the virus has potentially lethal effects on the cardiovascular system, major immune disturbances, as well as its adverse effects on the respiratory system shows very clearly the need for knowledge on the whole animal phenotype. These complex interactions between multiple body systems require carefully designed and controlled whole animal experimental studies.

The emphasis in modern medicine is now learning toward health throughout life and is now clear that adverse early events - even before birth - can greatly impair life-course health and shorten the lifespan. Therefore, the focus is moving toward maintaining a healthy span rather than expanding the life span ${ }^{5,13}$. A better knowledge of metabolic, cardiovascular, and neurological processes can also help improving health span in animals.

There is no doubt that studies performed with experimental animals have been gradually refined and improved, seeking better ways and methods, which allows reducing the number of animals to be used. Hence, the relevance of the joint work made by researchers and by the Institutional Animal Care and Use Committee (IACUC) to avoid the unjustified and indiscriminate use of animals.

\section{INSTITUTIONAL ANIMAL CARE AND USE COMMITTEE (IACUC) AT THE INSTITUTO NACIONAL DE CIENCIAS MÉDICAS Y NUTRICIÓN SALVADOR ZUBIRÁN}

The IACUC is composed by qualified staff scientist, most of them researchers at Instituto Nacional de Ciencias Médicas y Nutrición Salvador Zubirán (INCMNSZ) that are responsible of evaluating the feasibility and relevance of experimental research protocols, the rational use of animals, promoting animal welfare, supervising and endorsing animal procedures, as well as, reviewing the facilities where the animals are housed. All these in accordance with the NORMA Oficial Mexicana NOM-062-ZOO-1999 (Especificaciones técnicas para la producción, cuidado y uso de los animales de laboratorio) of the Mexican government and the International Guide for the care and use of laboratory animals ${ }^{4}$. At least one member of the IACUC must be a veterinarian who will advise the researcher with the necessary care for animals used in research studies, and at least another must be a member not affiliated with the institution in any way other than performing IACUC duties.

\section{THE MAIN FUNCTIONS OF THE INSTITUTIONAL ANIMAL CARE AND USE COMMITTEE AT INSTITUTO NACIONAL DE CIENCIAS MÉDICAS Y NUTRICIÓN SALVADOR ZUBIRÁN (FIG. 1)}

- Meeting every month and hold additional meetings when required 
Figure 1. The Institutional Animal Care and Use Committee (IACUC) in our Institute are composed by qualified staff scientist, who are responsible of evaluating the feasibility and relevance of experimental research protocols as well as the rational use of animals, promoting animal welfare, supervising and endorsing animal procedures, and reviewing the facilities where the animals are housed.

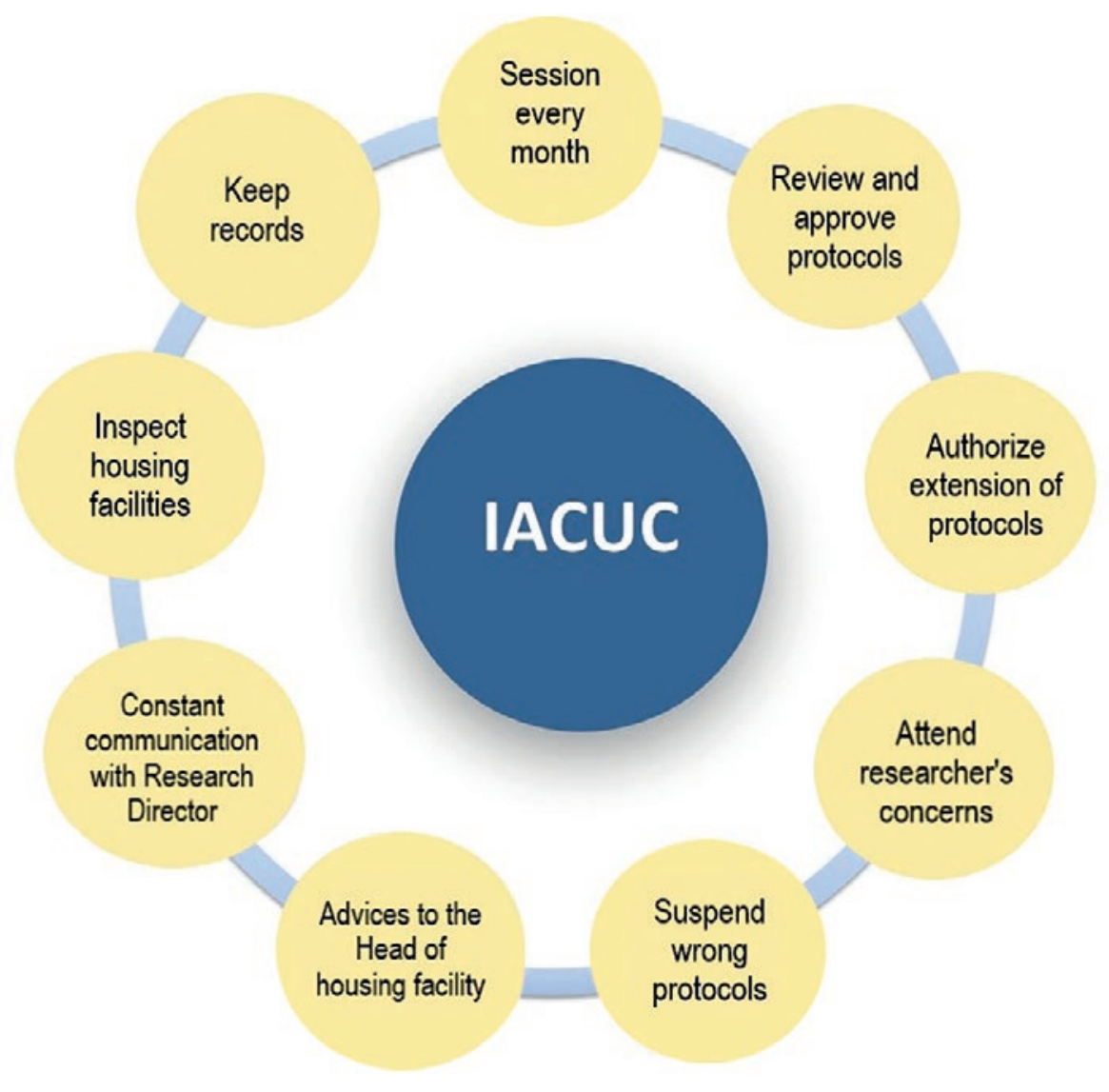

- Review and approve the proposed experimental protocols that involve the use of animals, as well as authorize any significant change in the proposals approved by IACUC that may arise during the development of the protocol

- Monitor that all the protocols submitted fulfill with the principle of the $3 \mathrm{R}$ 's: reduction, refinement, and replacement.

- When the studies are not completed in the time stipulated by the researcher, IACUC will review if an extension is appropriate

- Attend to researcher's concerns related to their protocol and procedures

- Reject protocols or interrupt active protocols when the experimental procedures are not carried out in accordance with the official Mexican STANDARD NOM-062-ZOO-1999 and the guide for the care and use of laboratory animals ${ }^{4}$

- Monitor the institutional program for the care and use of animals and respond to the concerns or complaints received

- Make recommendations to the Head of the Housing Facility at INCMNSZ regarding any aspect of the animal care and use program, equipment and installations, or about the training of personnel

- Resolve unexpected situations not considered in the Official Mexican STANDARD NOM-062-ZOO-1999, technical specifications for the production, care, and use of laboratory animals and the guide for the care and use of laboratory animals ${ }^{4}$ 
- Report to the Director of Research of any anomaly that arises with the reproduction or maintenance of animals, as well as any inappropriate conduct by users of the animal housing facility

- Verify the norms and guidelines established for the care and use of laboratory animals in accordance with the institutional requirements

- Inspect all areas of the animal housing facilities every 6 months.

- Keep the records of:

\section{Minutes of IACUC meetings}

2. Physical and digital files containing all the experimental protocols submitted for authorization by the IACUC and all the information related to each of them such as: acceptance letters, protocol modifications, extensions, protocol closures, and derived products from research.

3. Other communications or recommendations sent to the Research Director of the INCMNSZ.

\section{THE PRINCIPLE OF THE THREE R'S: REPLACEMENT, REDUCTION, AND REFINEMENT}

For a long time, there has been a general concern in the overall population as well as in the scientific community for the animal suffering experienced during research. Indeed, animals are living creatures that deserve respect and be treated with the highest ethical standards. This is what led William Russell and Rex Burch to publish a guide to maximally reduce animal pain in 195914. Since then, the concept of "applying humanitarian techniques in animal research" has been identified as the principles of the three R's: Replacement, Reduction, and Refinement. Nowadays and throughout the world, the 3R's are embedded in legislation ruling the use of animals for scientific purposes, such as Directive 2010/63/EU, which is the European Union legislation that protects animals used in research. In all these cases, the 3R's concept has become increasingly important, deriving in a plethora of scientific publications, networks, and organizations devoted to cover alternatives to animal experimentation ${ }^{15-19}$. Importantly, animal experiments that are unnecessary, use an excessively large number, are unreasonably painful or involve severe long-lasting pain which cannot be ameliorated, are defenseless, and should not be permitted by institutions ${ }^{20}$. Therefore, Animal Ethics Committees share responsibility for properly advise researchers aiming to work with animals, to adhere to the $3 R$ 's principles and improve the quality of the submitted protocols for better animal welfare, or if necessary, to reject these protocols in the case of insufficient justification to perform animal experimentation.

\section{Replacement}

Directive 2010/63/EU contains the following major clause: "An experiment shall not be performed if another scientifically satisfactory method of obtaining the result sought, not entailing the use of an animal, is reasonably and practicably available." Indeed, Russell and Burch first described animal replacement as any scientific method employing non-sentient material to replace the use of conscious living vertebrates in animal experimentation ${ }^{14}$. To date, Directive 2010/63/EU protects all live non-human vertebrates, including independently feeding larval forms and fetal forms of mammals from the last third of their normal development, as well as the invertebrate cephalopods such as octopus and squids (due to their well-developed nervous system and complex sensory organs). Replacement may be relative or absolute ${ }^{14}$. The first case considers the use of animals, but they should not be exposed to pain or distress during experimentation. Moreover, absolute replacement means that animals are not required at all at any stage of experimenta$\operatorname{tion}^{20}$. There is a wide range of well-established and emerging methodologies as alternatives for the absolute replacement of animals. Herein, we mention some of them that may be taken into consideration before choosing animal testing (Fig. 2).

\section{Cells/tissue cultures and biomaterials}

Culturing cells and tissues are maybe the easiest and lower cost option to exclude animal use in research. It involves the growth of cells/tissue outside the body under controlled conditions. A great advantage of cell and tissue cultures is that they are commercially available and a large number of different cell types from diverse organs from different species may be 
Figure 2. Schematic representation of the principle of the three R's: Replacement: in experimental research, look for well-established and emerging methodologies as alternatives for replacement of animals. Reduction: when replacement of animals is not possible, a decrease in the number of animals used in experimental protocols should be considered. Refinement: is a strategy to decrease in the incidence and/or severity of inhumane procedures applied to animals while participating in experimental protocols to minimize the pain and distress and to enhance welfare.

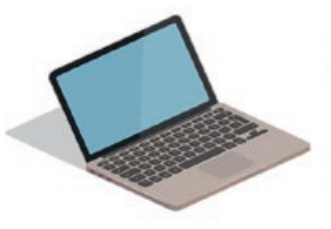
Computacional analysis

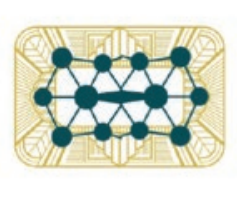

Organs on a chip

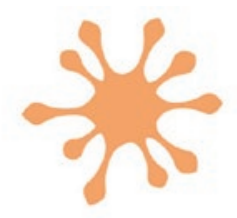

Organoids

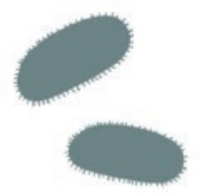

Prokaryote

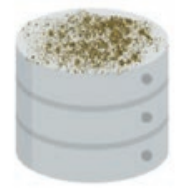

Cell cultures

\section{Replacement}

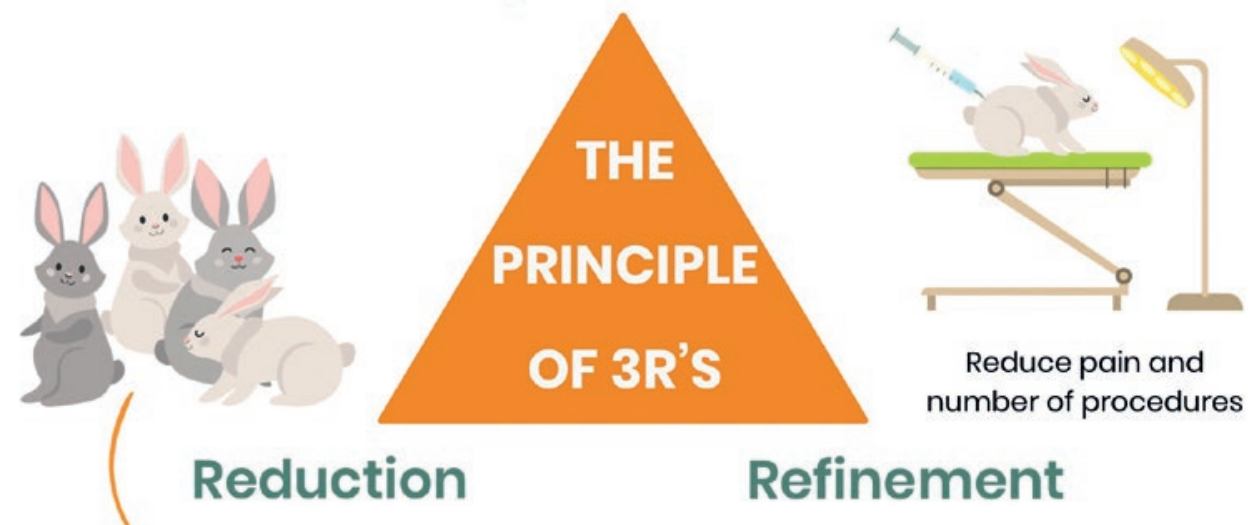

used, including pluripotent stem cells. Furthermore, commercially available engineered tissues may be found ${ }^{21}$. Cell cultures are routinely used for screening potential drugs or molecules to check their toxicity, efficacy, and bioactivity, as well as to study cell-cell interaction. Popular cellular and physiological processes studied in vitro include organogenesis, migration, cell proliferation, transdifferentiation, and regulation of signaling pathways, among others. Another recent approach for absolute replacement of animals is the use of biomaterials. Biomaterials are substances engineered to take a form which, alone or as part of a complex system, may be used in different applications, including research and the

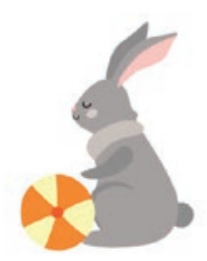

clinical practice. The fabrication of various tissues has been demonstrated through 3D bioprinting ${ }^{22,23}$. The 3D bioprinting systems use tissue-specific decellularized components of the extracellular matrix as bioinks to generate physiologically-relevant functional human tissues. This technique allows for the controlled assembly of cells and biomaterials to provide well-defined microstructures with specific biomechanical properties that recapitulate key features of native microenvironments, allowing intercommunications with the surrounding matrix to form a tissue-specific function that has proven to serve as powerful tools to study tissue homeostasis and even pathologies ${ }^{24}$. 


\section{Organoids culture}

Organoids are stem cell-derived three-dimensional, self-organizing microsystems that display an intact organ-like three-dimensional architecture and physiology with tissue-level functions and diverse disease phenotypes. They are actually considered as miniature organs grown in vitro, providing a wide range of possibilities for researchers ${ }^{25}$. Some of the advantages of organoids include that they are transplantable, improved versions of 2D models, and easy to manipulate. Organoids offer vast potential for investigating human development in health and disease, including advancing in precision and regenerative medicine. There is no doubt that organoids research is a promising field that will be of central importance in the decades to come, even though some disadvantages comprise difficulty to replicate and to integrate with other organ systems.

\section{Computational models}

Nowadays, computer associated technologies represent an easy and profitable strategy for alternatives to animal testing. For example, computer generated simulations and in silico modeling may be used to predict drug and molecular interactions, biological or toxic effects, and as well as bioactivity or pharmacological interactions. Moreover, potential alternative applications for currently approved drugs within a personalized medicine approach may also be accurately predicted ${ }^{26,27}$. There are many popular publicly and commercially available software packages that are useful as alternatives to animal testing based on mathematical tools (e.g., computer-aided drug design, structure-activity relationship [SARs], and quantitative SAR ${ }^{28}$. Of note, sharing the discovered data (like characteristics of excipients for a drug) and providing the whole databases greatly help to avoid the necessity of animal studies. In this regard, computational biology provides a powerful foundation to address critical scientific questions through modeling and theoretical exploration. Computational systems biology is a branch of this discipline that strives to generate a system-level understanding by analyzing available biological data using computational techniques $^{29}$. Computational modeling of biological systems is crucial to integrate and understand the continuously generated scientific data, being the Systems
Biology Markup Language (SBML), the most widely accepted open software platform for storing and exchanging models ${ }^{30}$. The SBML.org website includes a guide to many important software packages used in computational systems biology ${ }^{29}$. Furthermore, coupling in vitro/in silico data represent a powerful platform allowing accurate and mechanistic-level prediction of drug-induced effects. This is particularly important to study the liver, given that human liver metabolism differs from that of animals, and hepatotoxicity is always a risk when testing new drugs in the clinic. A good example of a computational-based model to address this issue would be VirtualLiver ${ }^{31}$, an in silico tool for predicting human liver drug toxicity, which couples equations describing the kinetics of biochemical pathways involved in liver homeostasis with those obtained from in vitro measurements quantifying various drug-induced perturbations. This informatics tool considers hepatotoxicity as the outcome of the interplay between different factors, including the patient genotype, the drugs currently prescribed, and underlying diseases ${ }^{28,29}$.

\section{Use of simpler organisms as substitutes for animals}

This is a rather logical alternative to animal testing and includes the use of prokaryotes, protists, fungi, and lower invertebrates. Unicellular microorganisms such as Escherichia coli and Saccharomyces cerevisiae are good examples of versatile and successful models for research: they have well-characterized genetic systems; they are inexpensive and rapidly growing. These and other microbes, including single-celled eukaryotes (protists) such as Dictyostelium discoideum have been used to understand fundamental aspects of biology, offering insights at the transcriptomic and proteomic level of cellular processes and functions. In particular, the amoeba $D$. discoideum has been used as a powerful genetic and cellular model for investigating host-pathogen interactions and microbial infections, mitochondrial diseases, and pharmacogenetic issues ${ }^{32}$. Regarding lower vertebrates, Danio rerio (zebrafish), due to its transparent characteristic, has been used in a wide array of internal anatomy studies. Its small size, short life cycle, and high fecundity also favor its laboratory use as a model for different biological processes, including the analysis of vertebrate development ${ }^{33}$. Examples of important invertebrates in research include: Amphimedon 
queenslandica (sea sponge), Aplysia sp. (sea slug), Caenorhabditis elegans (free-living, transparent nematode), Hydra sp. (marine Cnidaria), and Drosophila melanogaster (fruit fly), which have greatly contributed to breakthroughs in the scientific field involving genetics, neurology, evolution, developmental, regenerative biology, and even complex diseases as diabetes or cancer ${ }^{27,34}$.

\section{Organs-on-chips}

Organ chips are microfluidic cell culture devices containing microchannels that may be populated by living cells of diverse lineage and origin, allowing them to interact by sharing their respective secretomes and multicellular architectural characteristics, which ultimately conform a viable cellular network. This biosystem-on-a-chip recapitulates in vivo organ-level physiology and pathophysiology by recreating tissuelevel and organ-level structures and functions in vitro. The chips can be used instead of animals in disease/ physiology research, drug, and toxicity testing ${ }^{35-37}$.

\section{Reduction}

When the replacement of animals is not possible, reduction should be considered. Reduction means a decrease in the number of animals used in experimental protocols. Directive 2010/63/EU suggests that to reduce the number of animals, researchers should facilitate the establishment of programs for sharing the organs and tissues of the animals that are killed. This is a respectful way to make the most of the sacrificed animals, instead of just throwing away the unemployed tissues. Importantly, the welfare of animals used in scientific protocols highly depends on the staff's competence. Indeed, only adequately trained and competent individuals should manipulate laboratory animals, since unskilled persons may increase the risk of failed techniques or unwanted animal death, with the consequent increase in the number of animals required to obtain the desired result. By instance, surgeries to alter physiology (e.g., gonadectomy), transplantations, or simple routine methodologies such as retrieving a blood sample or administrating a drug (e.g., by cannula or intraperitoneally), may derive in the animal damage or death if performed without the proper expertise. Other strategies to reduce the number of animals include conducting pilot experiments (e.g., to determine the magnitude and variation of the effects of an experimental condition) and accurately designing the experimental protocol (e.g., in regards of sample size calculation and number of experimental groups needed). This is applicable if, by instance, the experimental protocol lacked an important control group, which is realized too late by the researcher, resulting in the need to repeat the whole experiment with more animals. The development of new clinical treatments is usually validated with preclinical animal testing, being the variability in the host response a common limitation. This is particularly true in studies of sepsis, which remains a prominent clinical problem. In the animal models, septic insults often generate a considerable variance across individuals, compelling to increase the sample size to ensure appropriate statistical power. This is why another way to reduce the number of animals in research is to decrease experimental variability, which may be achieved by conducting rigorous and modern protocols and techniques. In this regard, biotelemetry has proven to significantly reduce the number of mice needed to conduct sepsis experiments by quantifying host physiology in real-time ${ }^{38}$ (Fig. 2).

\section{Refinement}

When every effort has been made to reduce the number of animals for experimentation and replacement is not an alternative, refinement starts. Refinement refers to the decrease in the incidence and/or severity of inhumane procedures applied to animals while participating in experimental protocols to minimize the pain and distress and to enhance welfare. Russell and Burch defined refinement as: "simply to reduce to an absolute minimum the amount of distress imposed on those animals that are still used"14. Refinement also encompasses the responsibility of researchers to accurately design experimental protocols involving animals, given that an awkward design may result in wrong answers, waste of resources, and unnecessary suffering. There are a number of available guidelines that may help researchers to correctly plan their experimental protocols with animals, by instance: "PREPARE" which includes an easy "to do" checklist before starting working with animals ${ }^{39}$ (Fig. 2).

As Russell and Burch mentioned, almost any research question can be answered by a number of different procedures, and a skilled researcher will always choose 
"the most rapid, elegant, and simple one." Russell and Burch used as an example the study of a drug that is intended to reduce fear: instead of inducing fear by inflicting a terrifying experience to the pigeon (the animal chosen for the study), the bird could be trained to avoid visiting a platform by a very mild distressing experience of having found inaccessible food there. Then, the researcher may administer the drug and see whether the pigeon now spontaneously and without distress visited the forbidden platform, this strategy proved the effectiveness of the drug and avoided unnecessary suffering to the animal ${ }^{14}$. To compel researchers into refinement techniques, Russell and Burch used the argument made by Isaac Asimov: "Violence is the last refuge of the incompetent."

There are many strategies to minimize pain and distress in laboratory animals without compromising the scientific objectives. Herein, we mention some of them:

\section{Refinement of housing, handling conditions, and environmental enrichment (EE)}

Laboratory Animals welfare can be greatly improved by avoiding stress, with the advantage of obtaining more accurate results. Indeed, adequate handling conditions and housing, as well as EE, have a profound positive effect on research since animal distress can result in misleading results. The importance of $E E$ is well exemplified by a study of Cao et al. performed in a murine model of melanoma and colon cancer. This study housed a group of animals in large cages supplemented with running wheels, tunnels, igloos, huts, retreats, wood toys, labyrinths, and nesting material in addition to food and water, while the control group was kept in standard cages with only food and water. Results showed that even short-term exposure to EE improved resistance to cancer in mice and remarkably, serum from animals held under EE was able to inhibit cancer proliferation in vitro ${ }^{40}$. Systemic metabolic changes associated with EE included decreased serum insulin-like growth factor-1 and leptin levels.

Furthermore, refinement of housing conditions to lower distress is primordial after an experimental procedure or a surgery. For example, providing a quiet, warm place without intense illumination. A heating pad or lamp may be used to maintain body temperature and eye drops or ophthalmic ointment should be used to prevent desiccation or discomfort due to dry eyes when under anesthesia.

On the other hand, environmental factors in the animal facility (e.g., noises from air-conditioners and other electronic devices) challenge the biological balance of animals and may interfere with the animal's homeostasis and normal behavior. If the animal is unable to maintain its homeostasis, stress will develop in the course of time ${ }^{41}$. In line with this, staff working with animals should avoid the use of perfume, intense fragrances, and emission of loud sounds since animals are very sensitive to these stimuli that easily stress them. Care should also be taken while cleaning cages and transporting animals to diminish possible distressful situations.

\section{Blood sampling}

Refining blood sampling in mice, hamsters, and rats may be performed by puncture of the lateral saphenous vein, which is a less damaging alternative to retro-orbital or cardiac puncture. In addition, saphenous vein puncture allows rapid sampling, which if necessary can be repeated from the same site without the need for new puncture wounds.

\section{Use of analgesics}

Distress and pain may be alleviated/reduced by the use of painkillers. By instance, in the case of the immediate postoperative phase, analgesics must be used to mitigate the pain associated with surgical procedures.

\section{Avoid performing sacrifices and painful procedures in close presence of littermates}

Some animals such as rats and mice emit ultrasound vocalizations as a communicating strategy under stressful circumstances such as aggressive handling, fear, and pain. These sounds are not perceived by humans, but they certainly are by rodents. If the cages containing rats/mice are near the place where a sacrifice or a painful procedure is taking place, these animals may suffer distress due to the alarming signals emitted by the suffering animal. Moreover, the scent of blood is also a disturbing signal since it is 
associated with injury, danger, and death. Therefore, care should be taken to avoid this situation to prevent unnecessary distress of littermates.

\section{THE IACUC AND THE PRINCIPLE OF 3R'S}

Nowadays, researchers are more aware of the proper and respectful use of animals and resources. The IACUC helps to optimize the use of lab animals, ensuring that the experiments are carried out properly and in a humanitarian manner. One of the functions of the IACUC is precisely to monitor that all the protocols submitted fulfill with the principle of the 3R's. Therefore, the IACUC reviews the experimental design and justification for the adequate and restricted use of animals according to the calculation of the sample size. In the event that animals are used in excess, the researcher is encouraged to reduce the number of animals by refining the protocol, which is not approved until IACUC's observations are addressed. Furthermore, the experimental procedures are reviewed in detail to keep pain and suffering as low as possible. Likewise, when the research question can be answered with alternative experimental models such as cell cultures, computational analysis, or organoids, the IACUC suggests the replacement of animals by these means. The IACUC not only performs functions at the beginning of each research protocol but also has the power to withdraw permission from a protocol that does not promise sufficient benefit to offset the pain and distress caused in experimental animals or that do not adhere to the principle of the 3R's.

\section{CONCLUSIONS}

Experimental animals allow us to simulate significant diseases, which not only help to know and understand the mechanisms that trigger a pathophysiological process but also assist to evaluate physiological, physical, pharmacological, and biological treatments. Moreover, the evaluation of new treatments in experimental models helps to determine administration route, dose, immunotolerance, and toxicity. Along with this important progress, the need to provide training and guidance to the scientific community in all aspects related to experimental animals has become increasingly necessary. Animal research committees play a primary role in the evaluation of experimental research projects, from their feasibility to the rational use of animals, but above all, by monitoring animal welfare. It is increasingly important the awareness in the care of animals during research and the role of the veterinarian in this process, ensuring that it is possible to balance scientific objectives and animal welfare ${ }^{32,42}$. Although many laboratories apply the principle of the three 3 R's proposed by Russell and Burch $^{14}$, the number of animals used in the investigation has increased and the rational use of them must be well regulated. Animal experimentation is still necessary for certain teaching and research practices since there is still no sufficient technology to replace it altogether.

\section{ACKNOWLEDGEMENTS}

We would like to thank Dr. Yvett González Bobadilla for the edition of figures. Members of the Institutional Committee for the Care and Use of Animals: Lorenza Díaz and Elena Zambrano (Department of Reproductive Biology), Mariela Contreras (Department of Housing Facility), Jorge Barrios-Payán (Department of Pathology), Jose Carlos Crispín (Department of Rheumatology), César Bravo (Department of Experimental Surgery), Gabriela Alemán and Armando Tovar (Department of Physiology of Nutrition), Gerardo Gamba and Norma A. Bobadilla (Department of Nephrology), all from the Instituto Nacional de Ciencias Médicas y Nutrición Salvador Zubirán, Mexico City, Mexico, María Elena Flores (Instituto de Investigaciones Biomédicas) and Victor Julian Valdés (Instituto de Fisiología Celular), UNAM, Mexico City, Mexico, and Alejandra Armenta (external participant as an independent bioethicist)..

\section{REFERENCES}

1. Franco NH. Animal experiments in biomedical research: a historical perspective. Animals (Basel). 2013;3:238-73.

2. Animal research and the search for understanding. Nat Genet. 2006;38:497-8.

3. Conn PM, Parker J. Animal rights: reaching the public. Science. 1998;282:1417

4. National Research Council (U.S.) Committee for the Update of the Guide for the Care and Use of Laboratory Animals. Guide for the Care and Use of Laboratory Animals. Washington, DC: National Academy Press; 2011.

5. Taormina G, Ferrante F, Vieni S, Grassi N, Russo A, Mirisola MG. Longevity: lesson from model organisms. Genes (Basel). 2019; 10:518

6. World Medical Association. World medical association declaration of Helsinki: ethical principles for medical research involving human subjects. Bull World Health Organ. 2001;79:373-4. 
7. Dancet EA, Brannstrom M, Brasky K, Chai D, Chan AW, Conn PM, et al. The role of scientists and clinicians in raising public support for animal research in reproductive biology and medicine. Biol Reprod. 2013;88:33.

8. Phillips KA, Bales KL, Capitanio JP, Conley A, Czoty PW, Hart BA, et al. Why primate models matter. Am J Primatol. 2014; 76:801-27.

9. National Research Council (US) and Institute of Medicine (US) Committee on the Use of Laboratory Animals in Biomedical, Behavioral Research. Use of Laboratory Animals in Biomedical and Behavioral Research. Washington, DC: National Academy Press; 1988.

10. Norgaard M, Ehrenstein V, Vandenbroucke JP. Confounding in observational studies based on large health care databases: problems and potential solutions-a primer for the clinician. Clin Epidemiol. 2017;9:185-93.

11. Wooley JC, Lin H. Catalyzing Inquiry at the Interface of Computing and Biology. Washington, DC: National Academies Press; 2005.

12. Sabin $A B$. Present status of attenuated live-virus poliomyelitis vaccine. J Am Med Assoc. 1956;162:1589-96.

13. Halfon N, Larson K, Lu M, Tullis E, Russ S. Lifecourse health development: past, present and future. Matern Child Health J. 2014;18:344-65

14. Russell WM, Burch RL. The Principles of Humane Experimental Technique. London: Methuen; 1959

15. ALTEX-alternatives to Animal Experimentation. Available from: https://www.altex.org/index.php/altex

16. FRAME-researching Alternatives to Animal Testing. Available from: https://www.frame.org.uk.

17. The European Union Reference Laboratory for Alternatives to Animal Testing. Available from: https://www.ec.europa.eu/jrc/ en/eurl/ecvam

18. The PETA International Science Consortium Ltd. Alternative Methods Validated For Regulatory Use. Available from: https:// www.piscltd.org.uk/alternatives

19. The EURL ECVAM Search Guide Good Search Practice on Animal Alternatives. Available from: https://www.op.europa.eu/en/ publication-detail/-/publication/8835aa05-f780-454a-ac437752b38b394e.

20. Balls M. Replacement of animal procedures: alternatives in research, education and testing. Lab Anim. 1994:28:193-211.

21. Guerra L, Dellambra E, Panacchia L, Paionni E. Tissue engineering for damaged surface and lining epithelia: stem cells, current clinical applications, and available engineered tissues. Tissue Eng Part B Rev. 2009:15:91-112.

22. Murphy SV, Atala A. 3D bioprinting of tissues and organs. Nat Biotechnol. 2014;32:773-85.

23. Zhu W, Ma X, Gou M, Mei D, Zhang K, Chen S. 3D printing of functional biomaterials for tissue engineering. Curr Opin Biotechnol. 2016;40:103-12.
24. Yu C, Ma X, Zhu W, Wang P, Miller KL, Stupin J, et al. Scanningless and continuous 3D bioprinting of human tissues with decellularized extracellular matrix. Biomaterials. 2019;194:1-13.

25. Bredenoord AL, Clevers H, Knoblich JA. Human tissues in a dish the research and ethical implications of organoid technology. Science. 2017;355:eaaf9414.

26. Gottlieb A, Stein GY, Ruppin E, Sharan R. PREDICT: a method for inferring novel drug indications with application to personalized medicine. Mol Syst Biol. 2011;7:496.

27. Freires IA, Sardi JC, de Castro RD, Rosalen PL. Alternative animal and non-animal models for drug discovery and development: bonus or burden? Pharm Res. 2017;34:681-6.

28. Poloznikov A, Gazaryan I, Shkurnikov M, Nikulin S, Drapkina O, Baranova A, et al. In vitro and in silico liver models: current trends, challenges and opportunities. ALTEX. 2018;35:397-412.

29. Kitano H. Computational systems biology. Nature. 2002; 420:206-10

30. The Systems Biology Markup Language. Available from: http:// www.sbml.org/Main_Page.

31. The Virtual Liver. Available from: https://www.h-its.org/projects/virtual-liver.

32. Bozzaro S. The model organism Dictyostelium discoideum. Methods Mol Biol. 2013;983:17-37.

33. Winata CL, Korzh V. Zebrafish zic genes mediate developmental signaling. Adv Exp Med Biol. 2018;1046:157-77.

34. Doke SK, Dhawale SC. Alternatives to animal testing: a review. Saudi Pharm J. 2015;23:223-9.

35. Sontheimer-Phelps A, Hassell BA, Ingber DE. Modelling cancer in microfluidic human organs-on-chips. Nat Rev Cancer. 2019; 19:65-81.

36. Ronaldson-Bouchard K, Vunjak-Novakovic G. Organs-on-a-chip: a fast track for engineered human tissues in drug development. Cell Stem Cell. 2018;22:310-24.

37. Bhatia SN, Ingber DE. Microfluidic organs-on-chips. Nat Biotechnol. 2014:32:760-72.

38. Lewis A, Zuckerbraun B, Griepentrog J, Zhang X, Rosengart M. Reducing animal use with a biotelemetry-enhanced murine model of sepsis. Sci Rep. 2017;7:6622.

39. Smith AJ, Clutton RE, Lilley E, Hansen KE, Brattelid T. PREPARE: guidelines for planning animal research and testing. Lab Anim. 2018;52:135-41

40. Cao L, Liu X, Lin EJ, Wang C, Choi EY, Riban V et al. Environmental and genetic activation of a brain-adipocyte BDNF/ leptin axis causes cancer remission and inhibition. Cell. 2010; 142:52-64

41. Castelhano-Carlos MJ, Baumans V. The impact of light, noise, cage cleaning and in-house transport on welfare and stress of laboratory rats. Lab Anim. 2009;43:311-27.

42. Kehinde EO. They see a rat, we seek a cure for diseases: the current status of animal experimentation in medical practice. Med Princ Pract. 2013;22:52-61. 